\title{
Colonización y estructura de la comunidad de hongos micorrízicos arbusculares en Alsophila firma (Cyatheaceae) en bosque mesófilo de montaña en Veracruz, México
}

\author{
Luis Alberto Lara-Pérez ${ }^{1}$, Juan Carlos Noa-Carrazana ${ }^{1}$, Ángel de Jesús Landa López², Sergio \\ Hernández-González ${ }^{3}$, Iván Oros-Ortega ${ }^{1} \&$ Antonio Andrade Torres ${ }^{1 *}$ \\ 1. Instituto de Biotecnología y Ecología Aplicada, Universidad Veracruzana, Av. de las Culturas Veracruzanas No. 101, \\ Campus para la Cultura, las Artes y el Deporte, Col. Emiliano Zapata, C.P. 91090, Xalapa, Veracruz, México; \\ ingluislara@yahoo.com.mx, jnoa@uv.mx, ivanoros1109@hotmail.com, aandradet@gmail.com* \\ 2. Facultad de Ciencias Agrícolas, Universidad Veracruzana, Campus Xalapa. Circuito Universitario Gonzalo Aguirre \\ Beltrán s/n, Zona Universitaria, C.P. 91090, Xalapa, Veracruz, México; d-in-ho10@hotmail.com \\ 3. Facultad de Estadística e Informática, Universidad Veracruzana, Xalapa. Av. Xalapa esq. Manuel Ávila Camacho s/n, \\ C.P. 91020, Xalapa, Veracruz, México; shg5712@gmail.com \\ * Correspondencia
}

Recibido 06-II-2014. Corregido 10-VII-2014. Aceptado 13-VIII-2014.

\begin{abstract}
Colonization and structure of arbuscular mycorrhizal fungi community in Alsophila firma (Cyatheales: Cyatheaceae) from a tropical montane cloud forest in Veracruz, México. Alsophila firma is a tree fern that is distributed mainly in tropical montane cloud forest (TMCF) and is considered as a threatened species. Arbuscular mycorrrhizal fungi (AMF) have been proposed as an alternative in rescue programs of endangered species. However, our knowledge about diversity of AMF and mycorrhizal status of the species of TMCF is limited. In Mexico TMCF shows different degrees of conservation because of fragmentation and land use change. In this study, we evaluated the level of colonization, richness and abundances of spores of AMF in three fragments with different conservation status: conserved (100 years), secondary vegetation (17 years) and disturbed. For this, soil samples and roots were collected from five individuals of A. firma per site, with at least $100 \mathrm{~m}$ away from each other; a total of $100 \mathrm{~cm}$ of roots were analysed per site. Root samples showed AMF and occasionally dark septate fungi (DSF) colonizations. For the overall study, 19 species of AMF were recorded: Gigaspora (7), Acaulospora (4), Glomus (4), Funneliformis (2), Sclerocystis (1) and Scutellospora (1). The dominant species in the three sites were Funneliformis geosporum and Acaulospora scrobiculata. The highest diversity $\left(\mathrm{H}^{\prime}\right)$ and evenness $\left(\mathrm{J}^{\prime}\right)(\mathrm{p}<0.05)$ were found in the conserved site $\left(\mathrm{H}^{\prime}=1.7, \mathrm{~J}^{\prime}=0.66\right)$, when compared to the secondary vegetation $\left(H^{\prime}=1.5, J^{\prime}=0.61\right)$, and the disturbed site $\left(H^{\prime}=0.74, J^{\prime}=0.41\right)$. Statistical analysis showed that the AMF degree of colonization was significantly higher $(\mathrm{p}<0.05)$ in the conserved site; although, the disturbed site showed low richness and abundances of AMF, the degree of root colonization did not differ statistically $(\mathrm{p}<0.05)$ with the secondary vegetation site. Chao2 (Richness estimation model) showed that the number of analysed samples were sufficient to represent the structure of the AMF communities with values $>90 \%$. The present study confirmed that $A$. firma is a mycorrhizal species that exhibits high levels of colonization even in disturbed sites. We suggest that $F$. geosporum and $A$. scrobiculata may have the potential to inoculate the gametophyte and young sporophyte of $A$. firma, to support restoration programs, because of their abundances and high tolerance to disturbed sites. Rev. Biol. Trop. 62 (4): 1609-1623. Epub 2014 December 01.
\end{abstract}

Key words: tree fern, Glomeromycota, number of spores, diversity index, mycorrhizal colonization, disturbed, secondary and conserved vegetation.

En México hay 21 especies de helechos arborescentes en nueve géneros y seis familias (Mickel \& Smith, 2004; Korall, Pryer, Metzgar, Schneider, \& Conant, 2006; Smith et al.,
2006). La mayoría de estas especies tienen distribución en el bosque mesófilo de montaña (BMM), por lo que enfrentan una severa amenaza por la acelerada reducción de su hábitat 
(Williams, 1997; Vovides, Luna, \& Medina, 1997; Mickel, \& Smith 2004). Los helechos arborescentes mexicanos más vulnerables son las especies sustraídas para la obtención de maquique (masa de raíces adventicias), utilizado principalmente como ornamentos zoomórficos para jardines (Eleutério \& Pérez-Salicrup, 2006). Debido a la sobreexplotación, las poblaciones naturales han sido severamente dañadas y como consecuencia se encuentran protegidas por las leyes mexicanas (SEMARNAT, 2002). Entre las especies explotadas para este uso se encuentran: Alsophila firma, Cyathea divergens var. tuerckheimii, C. fulva y Sphaeropteris horrida (Bravo \& López, 1999; Eleutério, \& Pérez-Salicrup, 2006, 2009). Los individuos elegidos para la venta informal son helechos de gran talla, que incluyen hasta 107 helechos arborescentes adultos por año, para cubrir las exigencias del mercado (Eleutério \& PérezSalicrup, 2006).

La sustracción del maquique no sólo puede afectar las poblaciones naturales de los helechos arborescentes, sino también sus interacciones ecológicas. Por ejemplo, Alsophila firma tiene un crecimiento muy lento de aproximadamente $17.1 \pm 0.85 \mathrm{~cm}$ por año y alcanza la fertilidad aproximadamente a los nueve años (Mehltreter \& García-Franco, 2008). Tomando en cuenta la tasa de crecimiento, un individuo adulto de $10 \mathrm{~m}$ se estima que ha formado parte de la estructura vegetal por más de 60 años (Mehltreter \& García-Franco, 2008). Las tasas de extracción de troncos de $A$. firma para la venta informal, no sólo pueden reducir drásticamente el número de adultos fértiles en BMM (Eleutério \& Pérez-Salicrup, 2006), sino la riqueza de epífitas que incluyen a especies nativas como Trichomanes capillaceum (Mehltreter, FloresPalacios, \& García-Franco, 2005; Mehltreter \& García-Franco, 2008) y los microorganismos asociados en alguna etapa de su largo periodo de vida (Mehltreter, 2010).

Entender las interacciones ecológicas y factores abióticos que contribuyen a mejorar el establecimiento de los helechos arborescentes, puede proveer una base para desarrollar estrategias alternativas para contribuir a su conservación. Los hongos micorrízicos arbusculares (HMA) son uno de los componentes de la microbiota del suelo más importantes y extendidos de los ecosistemas naturales y de los sistemas de producción agrícola (Wang \& Qiu, 2006; Brundrett, 2009). La micorriza brinda mayor tolerancia a su hospedero frente a factores bióticos y abióticos adversos, debido a la mejora en la adquisición de nutrimentos, agua y protección contra patógenos (Newsham, Fitter, \& Watkinson, 1995; Smith \& Read, 1997). Por consiguiente, las plantas con micorriza tienen ventajas comparadas con las no micorrizadas al presentar mayores tasas de crecimiento foliar y radicular (Smith \& Read, 1997; Urgiles et al., 2009).

Se estima que el $90 \%$ de las plantas del mundo complementan su nutrición a través de la micorriza (Brundrett, 2009). El estatus micorrízico de los helechos arborescentes de la familia Cyatheaceae es confuso debido a que tiene especies ausentes de colonización, pero otras han sido reportadas como micorrízicas al presentar arbúsculos, que indican una simbiosis funcional (Cooper, 1976; Gemma, Koske, \& Flynn, 1992; Moteetee, Duckett, \& Russell, 1996; Zhao, 2000; Lehnert et al. 2009). Recientemente, se evidenció que Alsophila firma es una especie micorrízica que presenta cambios en los niveles de colonización y diversidad de esporas de HMA en lluvias y sequías en BMM (Lara-Pérez et al., 2014). Evidencias de trabajos en bosque tropical lluvioso indican que la mayoría de las especies arbóreas tienen asociaciones con HMA (Kottke, Beck, Oberwinkler, Homeier, \& Neill, 2004; Kottke et al., 2008). Sin embargo, se ha demostrado que hay diferentes comunidades de HMA en distintos hábitats y que en un mismo hábitat puede haber diferente composición de HMA en cada especie de hospedero (Hegalson, Daniell, Husband, Fitter, \& Young, 1998). Aunque se ha planteado que los HMA no son específicos de la planta que colonizan, diferentes aislados son más benéficos para algunos hospederos que otros (Van der Heijden, Boller, Wiemken, \& Sanders, 1998). La información del estatus micorrízico y los hongos involucrados en la 
interacción es clave para su aplicación en la reintroducción y restauración con especies nativas en el trópico (Schwartz et al., 2006; Urgiles et al., 2009). Debido a la fragmentación del BMM en México y el cambio de uso de suelo es necesario conocer la diversidad de HMA que se asocia a $A$. firma, tanto en bosques maduros como en bosques con diferente grado de conservación. De esta manera, se podrá conocer la abundancia y la riqueza de especies de HMA que permanecen al enfrentar disturbios en el ecosistema y el grado de colonización. El objetivo del presente trabajo fue evaluar el grado de colonización, riqueza y abundancia de esporas de HMA en la rizósfera de $A$. firma en tres fragmentos de BMM con diferente grado de conservación en Veracruz, México.

\section{MATERIALES Y MÉTODOS}

Área de estudio: Las muestras de raíz y suelo se recolectaron en tres reservas naturales localizadas en tres municipios en el centro y suroeste de Veracruz, México: 1) Reserva natural La Quinta Amatitla, en Zongolica, 2) Reserva natural La Martinica, en Banderilla y 3) La Reserva privada Las Cañadas, en Huatusco (Cuadro 1). Los sitios son fragmentos de BMM que difieren en su historia de manejo. Zongolica es un bosque maduro conservado, sin alteración por más de 100 años con acceso restringido; Huatusco es vegetación secundaria en recuperación de pastoreo de ganado vacuno por más de 17 años, con acceso restringido; y Banderilla, es un bosque alterado, en su mayoría vegetación secundaria y con acceso público para la práctica de senderismo. De aquí en adelante referidas únicamente por el municipio (Zongolica, Huatusco, Martinica). Las características físico-químicas del suelo se muestran en el cuadro 1.

Procedimiento de muestreo: En cada reserva se establecieron transectos a $300 \mathrm{~m}$ al interior del bosque y a lo largo del cauce del río o arroyo donde crece $A$. firma. En cada transecto se eligieron cinco helechos al azar, separados entre sí por al menos $100 \mathrm{~m}$ y se colectaron

CUADRO 1

Localización y características fisico-químicas de los tres sitios de estudio

TABLE 1

Localization and physic-chemical characteristics of the soil from the study sites

\begin{tabular}{|c|c|c|c|}
\hline & \multicolumn{3}{|c|}{ Sitios de estudio } \\
\hline & Zongolica & Huatusco & Banderilla \\
\hline Localización & 18039'07"71" N - 96059'49"57" W & 19¹1'23"14" N - 96059'11"74" W & 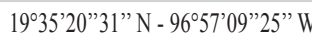 \\
\hline Elevación (msnm) & 1250 & 1300 & 1200 \\
\hline $\mathrm{CC} \%$ & 88.7 & 74.1 & 46.4 \\
\hline $\mathrm{CO} \%$ & 15.01 & 8.77 & 4.97 \\
\hline $\mathrm{DA}\left(\mathrm{g} / \mathrm{cm}^{3}\right)$ & 0.66 & 0.86 & 0.97 \\
\hline $\mathrm{P}(\mathrm{mg} / \mathrm{kg})$ & 13.8 & 3.6 & 2.3 \\
\hline $\mathrm{MO} \%$ & 25.88 & 15.12 & 8.57 \\
\hline $\mathrm{N} \%$ & 1.29 & 0.75 & 0.42 \\
\hline $\mathrm{pH}$ & 5.4 & 4.2 & 4.5 \\
\hline PMP\% & 58.7 & 46.6 & 33.1 \\
\hline Textura & Migajón arenoso & Migajón arenoso & Migajón arcillo arenoso \\
\hline
\end{tabular}

CC: Capacidad de campo, CO: Carbono orgánico, DA: Densidad aparente, P: Fósforo disponible, MO: Materia orgánica, N: Nitrógeno total, PMP: Punto de marchitez permanente.

CC: Field capacity, CO: Organic carbon, DA: Apparent density, P: Available phosphorus, MO: Organic matter, N: Total nitrogen, PMP: Permanent wilting point. 
muestras de suelo y raíces en los meses de agosto-septiembre 2012. Las muestras de raíces y suelo se obtuvieron de los cuatro puntos cardinales de cada individuo. En cada punto cardinal se eligió una raíz y se desenterró hasta los $35-40 \mathrm{~cm}$ desde la base del helecho a una profundidad de $4-10 \mathrm{~cm}$, y se tomaron entre $7-10 \mathrm{~cm}$ de fragmentos de raicillas. Las raíces se fijaron en alcohol al $70 \%$ hasta su procesamiento en laboratorio. Después de colectar las raicillas, se colectaron $100 \mathrm{~g}$ de suelo en cada punto cardinal a una profundidad de $15-25 \mathrm{~cm}$. Los $400 \mathrm{~g}$ de suelo colectado de cada individuo se homogenizaron y transportaron al laboratorio en bolsas negras de polietileno y se almacenaron a $4^{\circ} \mathrm{C}$ hasta su procesamiento.

Clareo y tinción: Se realizó el clareo y tinción con la metodología de Phillips y Hayman (1970) modificada. Se eligieron secciones de raíces de $1.5-2.5 \mathrm{~cm}$ de largo, se aclararon con $\mathrm{KOH}$ al $10 \%(\mathrm{p} / \mathrm{v})$ hasta por $60 \mathrm{~min}$ en autoclave a $120^{\circ} \mathrm{C}\left(14-15 \mathrm{lb} / \mathrm{pulg}^{2}\right.$ de presión de vapor). Cuando fue necesario, se utilizó una solución alcalina de $\mathrm{H}_{2} \mathrm{O}_{2}$ al $3 \%(11 \mathrm{~V})$ por 20 min a temperatura ambiente, para terminar de blanquear las raíces. Después las muestras se enjuagaron y se acidificaron con $\mathrm{HCl}$ al $1 \%$ $(\mathrm{v} / \mathrm{v})$ por $1 \mathrm{~h}$. El teñido se realizó con azul de tripano en lactoglicerol (glicerol al $31 \% \mathrm{v} / \mathrm{v}$, ácido láctico al $31 \% \mathrm{v} / \mathrm{v}$ y azul de tripano al $0.05 \% \mathrm{p} / \mathrm{v}$ ) por tres minutos en autoclave a $120^{\circ} \mathrm{C}$. Las raíces teñidas se almacenaron en lactoglicerol hasta su análisis.

Estimación de la colonización micorrízica: Las raíces teñidas de cada individuo se montaron por duplicado en portaobjetos con lactoglicerol, distribuyendo 10 fragmentos de aproximadamente $1 \mathrm{~cm}$ por cada portaobjetos para analizarse en un microscopio compuesto (Leica DM1000). Por cada sitio se analizaron un total de 10 preparaciones equivalentes a $100 \mathrm{~cm}$ de raíz. La colonización se evaluó utilizando la metodología de cuantificación múltiple (MQM; Ormsby et al., 2007; Kaminskyj, 2008) modificada de McGonigle et al. (1990). Para cada individuo se analizaron aproximadamente 150 intersecciones separadas a la misma distancia (1-2mm) inspeccionando con los objetivos 20x, 40x y 100x. En la intersección se registraron por separadado: hifas aseptadas típicas de HMA, vesículas, arbúsculos e hifas intracelulares enrolladas similares a ovillos (coils). Para la clasificación de morfología de colonización se consultaron los trabajos de Dickson, (2007) y Kubota et al. (2005). Para los hongos septados oscuros se consideraron las hifas septadas café y/o hialinas (Jumpponen \& Trappe, 1998). Las estructuras micorrízicas encontradas se documentaron con una cámara digital (Sony Cyber-shot DSC-W320). Las preparaciones de esporas y raíces teñidas se depositaron como material de referencia en el Instituto de Biotecnología y Ecología Aplicada (INBIOTECA-UV).

Extracción e identificación de esporas de HMA: Para el aislamiento de esporas se utilizaron $10 \mathrm{~g}$ se suelo seco con la técnica de tamizado húmedo y decantación (Gerdemann $\&$ Nicolson, 1963), seguida por una centrifugación en sacarosa (Daniels \& Skipper, 1982) de acuerdo con la modificación propuesta por Oehl et al. (2003). Las esporas que lucían intactas y saludables se consideraron en el conteo y se agruparon de acuerdo a sus características morfológicas. Se realizaron preparaciones permanentes con al menos 20 esporas montadas con alcohol polivinílico en lactoglicerol (PVLG) y reactivo de Melzer en PVLG 1:1 (v/v) (Morton, 1993). Las características fenotípicas se compararon con las descripciones originales (Schenck \& Pérez, 1990) y referencias en línea de la descripción de especies del INVAM de West Virginia University, USA (http://invam.caf.wvu.edu/) y del Department of Plant Pathology, University of Agriculture in Szczecin, Poland (http://www.agro.ar.szczecin.

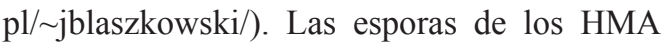
se midieron con un micrómetro de ocular y el software Image J (ImageJ 1.40; US NationalInstitutes of Health, Bethesda, MD11).

La colonización micorrízica y la abundancia de esporas se analizaron mediante una prueba Kruskal-Wallis $(\mathrm{p}<0.05)$. El índice de 
diversidad de Shannon-Wiener (H') se calculó de acuerdo a la fórmula: $\mathrm{H}^{\prime}=-\sum\left(P_{i} \ln \left[P_{i}\right]\right)$. Donde $P_{i}$ es la abundancia relativa, $\ln$ es el logaritmo natural. El índice de equitatividad de Pielou (J') se obtuvo con la ecuación: J' $=\mathrm{H}^{\prime} /$ $\log (\mathrm{S})$, donde $\mathrm{S}$ es el número total de especies aisladas en cada sitio. El índice de dominancia de Simpson (D) por la fórmula: $\mathrm{D}=\sum P_{i}^{2}$. Para el análisis de los datos se utilizó el programa PAleontological STatistics (PAST) (Hammer et al. 2001). Para estimar diferencias en los índices de Shannon-Wiener y de Simpson entre sitios se realizó un ANOVA de una vía seguida de una prueba de Tukey $(\mathrm{p}<0.05)$. Para hacer un análisis de la riqueza de especies se aplicó el algoritmo del Programa EstimateS 8.0 (Colwell, 2006), utilizando el modelo Mao-Tau con riqueza y abundancia de las especies en cada sitio y de todo el estudio. El orden de las muestras fue aleatorizado por 100 repeticiones. El esfuerzo de muestreo fue evaluado por medio del estimador no-paramétrico de diversidad Chao 2 (Magurran, 2004).

\section{RESULTADOS}

Colonización micorrízica: Los HMA estuvieron presentes en 26 de 30 preparaciones de raíz de $A$. firma. Las estructuras de los HMA registradas fueron hifas, hifas extraradicales, arbúsculos, coils y vesículas (Fig. 1 A-F). En Banderilla dos individuos no presentaron colonización de ningún tipo. Los hongos septados oscuros (HSO) sólo estuvieron presentes en las muestras de Zongolica y Huatusco con porcentajes menores a cinco por ciento (Cuadro 2). La colonización de HSO fue por hifas hialinas finas y microesclerocios (Fig. $1 \mathrm{G}-\mathrm{H}$ ).

La colonización micorrízica inicia por la epidermis de la raíz evidenciada por la presencia de hifopodios (Fig. 1A). El tipo de colonización en todas las muestras fue de tipo Paris (Fig. 1 D-F). El porcentaje promedio de arbúsculos y coils fue diferente $(\mathrm{p}<0.05)$ en todos los sitios. La colonización por hifas y colonización total de raíz fue menor $(\mathrm{p}<0.05)$ en Banderilla a comparación con Zongolica y Huatusco, donde no se presentaron diferencias significativas (Cuadro 2). No se observó diferencia significativa en el porcentaje de vesículas e hifas septadas entre sitios. Los porcentajes mayores de colonización total se presentaron en Zongolica seguida de Huatusco y Banderilla (Cuadro 2). La colonización micorrízica en Banderilla fue menos consistente y presentó amplia variabilidad en el nivel de colonización (Cuadro 2).

Riqueza y abundancia de HMA: En total se aislaron 1521 esporas y esporocarpos de HMA de 15 muestras de suelo de la rizósfera de A. firma en tres fragmentos de BMM en las que se encontraron 19 morfoespecies (Fig. 2 y Cuadro 3). Diez de los morfotipos se lograron identificar a especie y el resto sólo a género (Cuadro 3). Los géneros más abundantes fueron Gigaspora (7), Acaulospora (4), Glomus (4), Funneliformis (2), Sclerocystis (1) y Scutellospora (1). El número de especies de HMA aisladas por individuo variaron en los diferentes sitios. Zongolica presentó mayor número de especies por individuo en $10 \mathrm{~g}$ de suelo seco (7-12), Huatusco presentó de 7-10 especies y Banderilla registró el menor número de especies por individuo (2-6). La riqueza total de HMA por sitio se presentó en el mismo orden de prioridad Zongolica (13), Huatusco (11), y Banderilla (6). La abundancia de esporas varió significativamente $(\mathrm{p}<0.05)$ entre los sitios (Cuadro 3), presentando mayor abundancia Zongolica que es el sitio con bosque conservado, mientras que la menor abundancia se registró en Banderilla. Basados en la abundancia relativa las especies dominantes en los tres sitios fueron: Funneliformis geosporum y Acaulospora scrobiculata. Las especies Funneliformis geosporum, Acaulospora scrobiculata, Gigaspora decipiens, Funneliformis constrictum y Gigaspora sp. 1 estuvieron presentes en los tres sitios con baja abundancia relativa (Cuadro 3).

La diversidad (H') de HMA en la rizósfera de A. firma fue estadísticamente diferente para cada sitio (Cuadro 4). Zongolica presentó la mayor diversidad de HMA, y la menor diversidad se registró en Banderilla (Cuadro 4). La 


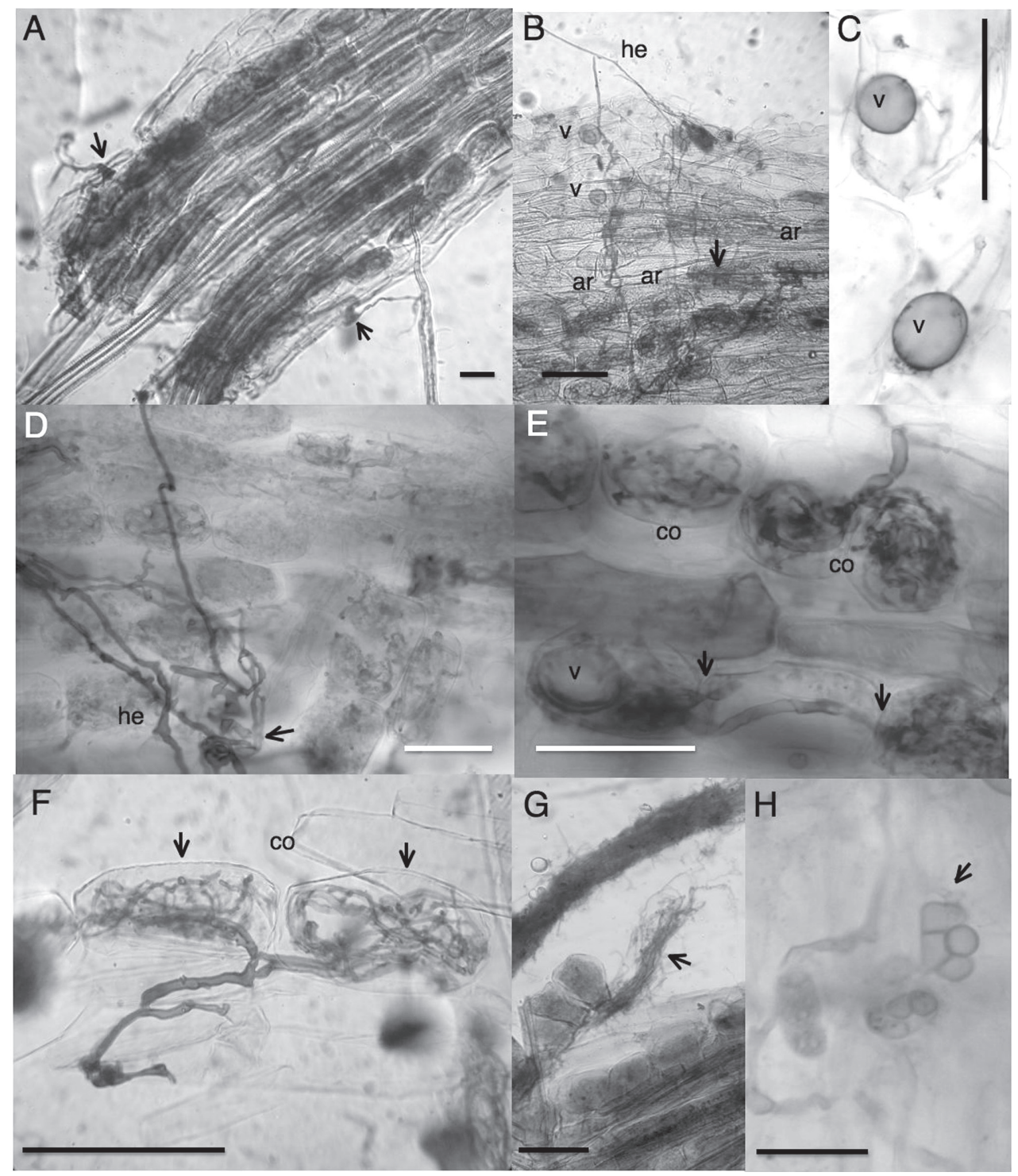

Fig. 1. Colonización de hongos micorrízicos arbusculares en raíces de Alsophila firma. (A) Fragmento de raíz densamente colonizado por arbúsculos con puntos de entrada (hifopodios) indicados por las flechas, (B) hifa extraradical y colonización por vesículas y arbúsculos en las células corticales, (C) vesículas, (D) hifas extraradicales y colonización tipo Paris, (E) colonización intercelular (flechas), vesícula y coils, (F) coils, (G) colonización por hongos septados oscuros, (H) microesclerocio. he: hifa extraradical, ar: arbúsculo, v: vesícula. bar: $100 \mu \mathrm{m}$.

Fig. 1. Arbuscular mycorrhizal fungi colonization within the roots of Alsophila firma. (A) Root fragment densely colonized by arbuscule with penetration points (hyphopodia) showed by arrows, (B) extraradical hyphae and colonization by vesicles and arbuscules in cortical cells, (C) vesicles, (D) extraradical hyphae and Paris-type colonization, (E) vesicle and hyphal coils, (F) hyphal coils, $(\mathbf{G})$ dark septate fungi colonization, $(\mathbf{H})$ microsclerotia. he: extraradical hyphae, co: coil, v: vesicle. bar: $100 \mu \mathrm{m}$. 
CUADRO 2

Valores promedios $( \pm \mathrm{DE}$ ) del porcentaje de la abundancia de estructuras de hongos micorrízicos en raíces de Alsophila firma en tres fragmentos de bosque mesófilo de montaña

TABLE 2

Mean values $( \pm \mathrm{SD})$ of the percent abundance of mycorrhizal fungal structures within the roots of Alsophila firma in three fragments of tropical montane cloud forest

\begin{tabular}{lcccccc}
\multicolumn{1}{c}{ Sitios } & $\mathrm{H}$ & $\mathrm{V}$ & $\mathrm{A}$ & $\mathrm{C}$ & HS & Col. total \\
Zongolica & $52.4 \pm 9.5^{\mathrm{a}}$ & $0.12 \pm 0.21^{\mathrm{a}}$ & $32.5 \pm 11.7^{\mathrm{a}}$ & $2.65 \pm 1.2^{\mathrm{a}}$ & $0.18 \pm 0.18^{\mathrm{a}}$ & $55.35 \pm 10.11^{\mathrm{a}}$ \\
Huatusco & $39.7 \pm 10.1^{\mathrm{a}}$ & $1.4 \pm 3.4^{\mathrm{a}}$ & $19.5 \pm 6.5^{\mathrm{b}}$ & $4.2 \pm 1.8^{\mathrm{b}}$ & $1.2 \pm 3.6^{\mathrm{a}}$ & $46.6 \pm 9.8^{\mathrm{a}}$ \\
Banderilla & $21.6 \pm 23.1^{\mathrm{b}}$ & $0 \pm 0^{\mathrm{a}}$ & $11.2 \pm 10^{\mathrm{c}}$ & $2 \pm 4.9^{\mathrm{c}}$ & $0 \pm 0^{\mathrm{a}}$ & $23.6 \pm 23.8^{\mathrm{b}}$ \\
\hline
\end{tabular}

Letras diferentes en las columnas muestran diferencias significativas $(\mathrm{p}<0.05)$ entre los sitios utilizando la prueba de Kruskal-Wallis.

DE: Desviación estándar, H: Hifa, V: Vesículas, A: Arbúsculos, C: Coils, HS: Hifas septadas, Col. Total: Colonización Total.

Different letters within columns showed significant differences $(\mathrm{p}<0.05)$ between sites using Kruskal-Wallis test.

SD: Standard deviation, H: Hyphae, V: Vesicle, A: Arbuscules, C: Coils, HS: Septate hyphae, Col. Total: Total colonization.

\section{CUADRO 3}

Número de esporas, riqueza y abundancia relativa de los HMA presentes en la rizósfera de Alsophila firma

TABLE 3

Number of spore, richness and relative abundance of arbuscular mycorrhizal fungi of the Alsophila firma rhizosphere

\begin{tabular}{|c|c|c|c|c|c|c|}
\hline Especies & Zongolica & AR & Huatusco & $\mathrm{AR}$ & Banderilla & $\mathrm{AR}$ \\
\hline Acaulospora sp. 1 & 211 & 0.325 & 77 & 0.107 & 0 & 0 \\
\hline Acaulospora scrobiculata & 190 & 0.293 & 253 & 0.352 & 18 & 0.115 \\
\hline Funneliformis geosporum & 133 & 0.205 & 292 & 0.407 & 125 & 0.801 \\
\hline Gigaspora sp. 2 & 37 & 0.057 & 25 & 0.034 & 1 & 0.006 \\
\hline Glomus sp. 1 & 18 & 0.027 & 0 & 0 & 0 & 0 \\
\hline Gigaspora decipiens & 17 & 0.026 & 3 & 0.004 & 3 & 0.019 \\
\hline Acaulospora sp. 2 & 10 & 0.015 & 0 & 0 & 0 & 0 \\
\hline Gigaspora sp. 1 & 9 & 0.013 & 0 & 0 & 0 & 0 \\
\hline Gigaspora sp. 3 & 9 & 0.013 & 0 & 0 & 0 & 0 \\
\hline Funneliformis constrictum & 6 & 0.009 & 13 & 0.018 & 3 & 0.019 \\
\hline Gigaspora sp. 4 & 5 & 0.007 & 0 & 0 & 0 & 0 \\
\hline Gigaspora sp. 5 & 2 & 0.003 & 0 & 0 & 0 & 0 \\
\hline Glomus intraradices & 1 & 0.001 & 0 & 0 & 0 & 0 \\
\hline Acaulospora mellea & 0 & 0 & 2 & 0.002 & 0 & 0 \\
\hline Gigaspora albida & 0 & 0 & 16 & 0.022 & 0 & 0 \\
\hline Glomus microaggregatum & 0 & 0 & 0 & 0 & 6 & 0.038 \\
\hline Glomus sp. 2 & 0 & 0 & 3 & 0.004 & 0 & 0 \\
\hline Sclerocystis coremioides & 0 & 0 & 5 & 0.006 & 0 & 0 \\
\hline Scutellospora sp. 1 & 0 & 0 & 28 & 0.039 & 0 & 0 \\
\hline Total de especies & 13 & & 11 & & 6 & \\
\hline
\end{tabular}

AR: Abundacia relativa. / AR: Relative abundance. 


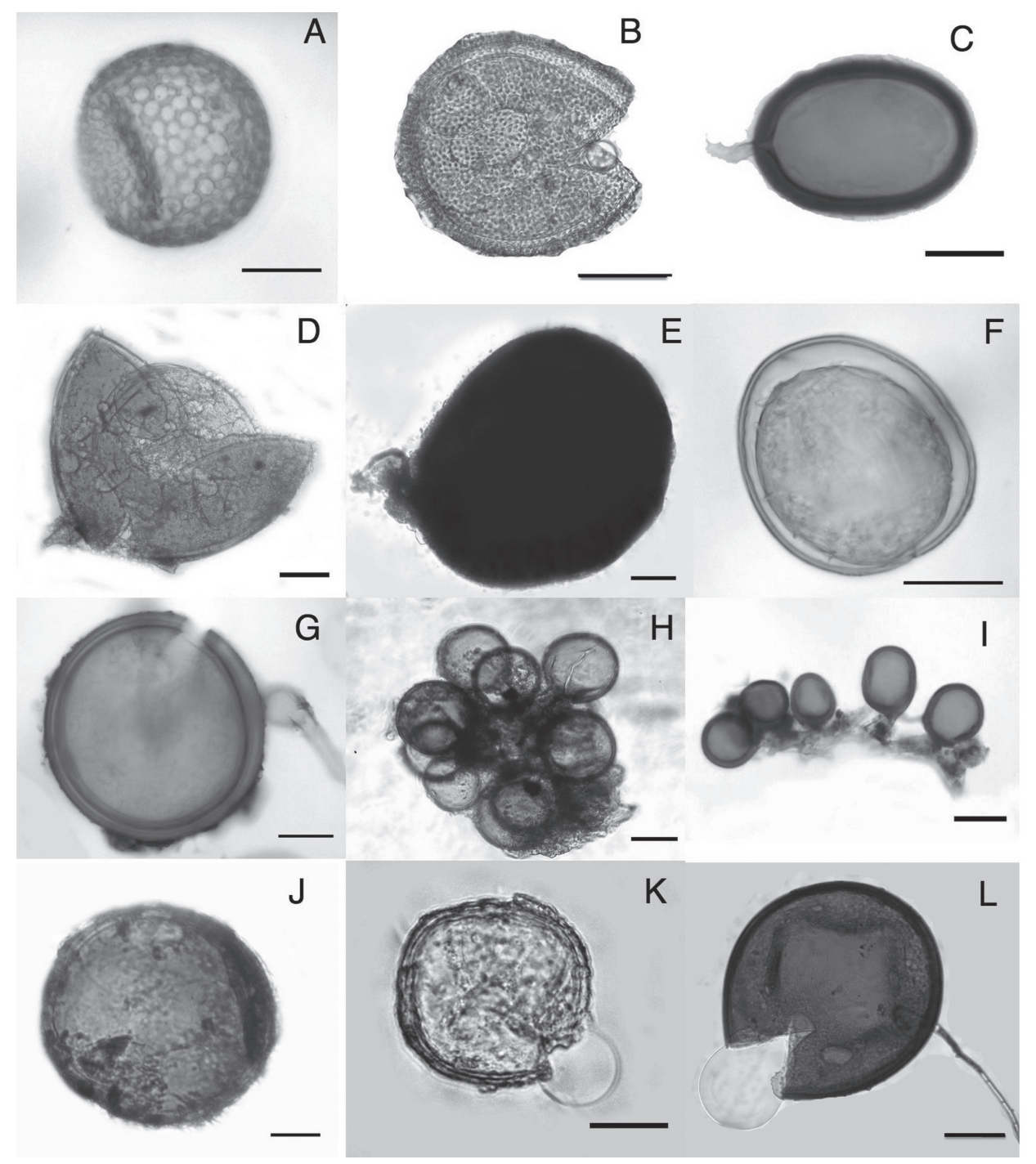

Fig. 2. Esporas de hongos micorrízicos arbusculares encontrados en la rizósfera de Alsophila firma en las reservas naturales de bosque mesófilo de montaña en Veracruz, México: Huatusco, Banderilla y Zongolica. (A) Acaulospora sp. 1 (B) Acaulospora scrobiculata, (C) Funneliformis geosporum, (D) Gigaspora sp. 2, (E) Funneliformis constrictum, (F) Acaulospora mellea, (G) Gigaspora albida, (H) Sclerocystis coremioides (I) Glomus microaggregatum, (J) Scutellospora

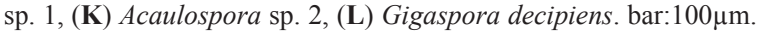

Fig. 2. AMF spores isolated from the rhizosphere of Alsophila firma in natural reserves of tropical montane cloud forest: Huatusco, Banderilla y Zongolica. (A) Acaulospora sp. 1 (B) Acaulospora scrobiculata, (C) Funneliformis geosporum, (D) Gigaspora sp. 2, (E) Funneliformis constrictum, (F) Acaulospora mellea, (G) Gigaspora albida, (H) Sclerocystis coremioides (I) Glomus microaggregatum, (J) Scutellospora sp. 1, (K) Acaulospora sp. 2, (L) Gigaspora decipiens. bar: $100 \mu \mathrm{m}$. 
CUADRO 4

Medidas de diversidad de HMA en los sitios de estudio

TABLE 4

Diversity measurements of AMF communities in the study sites

\begin{tabular}{lcccccc}
\multicolumn{1}{c}{ Sitios de muestreo } & Riqueza específica & Chao 2 & D & E & H' & Abundancia \\
Todos los sitios & 19 & 20 & - & - & - & 1521 \\
Zongolica & 13 & 14 & $0.24^{\mathrm{a}}$ & 0.66 & $1.7^{\mathrm{a}}$ & $648^{\mathrm{a}}$ \\
Huatusco & 10 & 10 & $0.30^{\mathrm{b}}$ & 0.61 & $1.5^{\mathrm{b}}$ & $717^{\mathrm{a}}$ \\
Banderilla & 6 & 6 & $0.65^{\mathrm{c}}$ & 0.41 & $0.74^{\mathrm{c}}$ & $156^{\mathrm{b}}$ \\
\hline
\end{tabular}

Letras diferentes indican diferencia significativa. Abundancia (Kruskal-Wallis, $\mathrm{p}<0.05$ ). Índice de dominancia de Simpson $\mathrm{e}$ índice de diversidad de Shannon-Wiener (Prueba de Tukey después de un ANOVA de una vía, $\mathrm{p}<0.05$ ).

D: Índice de dominancia de Simpson, E: Equitatividad, H’: Índice de diversidad de Shannon-Wiener.

Different letters showed significant differences. Abundance (Kruskal-Wallis test, $\mathrm{p}<0.05$ ). Simpson's index of dominance and Shannon-Wiener's index of diversity (Tukey's test after a one-way ANOVA, $p<0.05$ ).

D: Simpson's index of dominance, E: Evenness, H': Shannon-Wiener's index of diversity.

distribución de las especies fue más uniforme en Zongolica $\left(\mathrm{J}^{\prime}=0.66\right)$, en comparación con Huatusco $\left(\mathrm{J}^{\prime}=0.61\right)$ y Banderilla $\left(\mathrm{J}^{\prime}=0.41\right)$, donde se refleja mayor dominancia de especies $(\mathrm{D}=0.65)$ (Cuadro 4).

Las curvas de acumulación de especies demuestran que la cantidad de muestras analizadas refleja la estructura general de la comunidad de HMA. El estimador no paramétrico de riqueza de especies de Chao 2 reporta un 100\% para Huatusco y Banderilla y $93 \%$ en Zongolica (Cuadro 4). La riqueza total estimada por el índice fue de 20 y se registró un total de 19 especies en todo el estudio (Cuadro 4).

\section{DISCUSIÓN}

El presente trabajo indica que $A$. firma presenta diferentes niveles de colonización de estructuras micorrízicas y diversidad de esporas en tres fragmentos de BMM con diferente grado de conservación. La colonización de $A$. firma fue principalmente por HMA y de manera secundaria por HSO. La riqueza de esporas en la rizósfera de $A$. firma incluye 19 especies de HMA en los diferentes sitios. Sin embargo, las especies Acaulospora scrobiculata, Funneliformis geosporum fueron las mejor representadas en los tres sitios de BMM. La mayor riqueza y abundancia de HMA se registró en el bosque con mayor grado de conservación (Zongolica), sin embargo, en el bosque secundario (Huatusco) y el bosque con más actividad antrópica (Banderilla), aunque la riqueza y abundancia de HMA son menores, se observó alta colonización micorrízica.

La colonización de A. firma fue principalmente por HMA como se ha reportado en los trabajos de asociación micorrízica de helechos y licopodios (Wang \& Qui, 2006; Kessler et al., 2009; Brundrett, 2009; Zubek et al., 2010). Aunque, la presencia de HSO ha sido reportada para varias especies de helechos, este es el primer registro para A. firma (Jumpponen \& Trappe, 1998; Lehnert et al., 2009; Zubek et al., 2010), y sólo hay un registro de HSO en otra especie de la familia Cyatheaceae (Jumpponen \& Trappe, 1998). No obstante, en 25 especies de helechos arborescentes estudiadas de las familias Cyatheaceae, Cibotiaceae, Dicksoniaceae no hay registros de colonización por HSO (Cooper, 1976; Gemma et al., 1992; Moteetee et al., 1996; Zhao, 2000; Lara-Pérez, 2007; Lehnert et al., 2009).

Los estudios del estatus micorrízico en helechos arborescentes son inconsistentes, ya que los niveles de colonización varían de 0 a >75\% (Zhao, 2000; Lara-Pérez, 2007; Lehnert 
et al., 2009; Moteetee et al., 1996, Cooper, 1976; Gemma et al., 1992). Incluso en otros estudios, especies del mismo género Alsophila gigantea, A. podophylla, A. conantiana y A. dregei no registraron colonización micorrízica (Zhao, 2000; Moteetee et al., 1996). Recientemente, se demostró que $A$. firma es una especie micorrízica y que el grado de colonización varía en función de la estaciónalidad (Lara-Pérez et al., 2014). En este trabajo, se demuestra que $A$. firma presenta colonización con presencia de arbúsculos independientemente del grado de conservación del sitio en que se encuentre, lo que podría indicar la funcionalidad en la nutrición bidireccional (Brundrett, 2009). Aunque la colonización por arbúsculos estuvo presente en los tres sitios estudiados la frecuencia fue más homogénea en Zongolica con respecto a Huatusco y Banderilla, donde la actividad antrópica es mayor. En Banderilla se registraron cuatro muestras correspondientes a dos individuos, los cuales no presentaron evidencia de colonización destacando la importancia de incrementar el número de muestras en sitios alterados, como lo sugiere Brundrett (2009).

Los índices de diversidad y las curvas de acumulación de especies demuestran que la diversidad fue mayor en Zongolica, que es el sitio más conservado con respecto a Huatusco y Banderilla. Aunque el presente estudio sólo midió la riqueza y abundancia de los HMA en la rizósfera de $A$. firma, los resultados son similares a los reportados en estudios de análisis de secuencias, donde la riqueza de ribotipos y morfotipos de HMA es más baja en ecosistemas con mayor impacto antropogénico (Helgason et al., 1998; Oehl et al., 2003; Öpik et al., 2006). Sin embargo, en un bosque tropical lluvioso de Ecuador, se demostró que la riqueza de secuencias de grupos taxonómicos de HMA, no difiere en ecosistemas prístinos en comparación con ecosistemas degradados, pero si en su estructura (Haug et al., 2010). De manera opuesta, la riqueza específica basada en morfoespecies de esporas de HMA resultó mayor tras la conversión de BMM a cafetales con diferentes niveles de sombra (Arias, Heredia-Abarca, \& Sosa, 2011), asimismo en la conversión de BMM a milpa (Violi et al. 2008). Sin embargo, la estructura de los HMA contrasta en los diferentes ecosistemas (Violi et al. 2008; Arias et al., 2011). Si bien la diversidad de esporas en el presente estudio fue significativamente mayor en Zongolica, los sitios en recuperación y alterado albergan una alta riqueza de plantas que pueden estar asociados a diferentes especies de HMA como se ha demostrado con análisis de filotipos en ecosistemas de bosques de montaña similares (Kottke et al., 2004; 2008). Además, las evidencias de los estudios moleculares sugieren que la verdadera diversidad es de alguna manera más alta comparada con la riqueza morfológica (Öpik, et al., 2009). Considerando lo anterior, es probable que la riqueza de especies estimada mediante la morfología de esporas aún no representa la riqueza total de los sitios y ésta puede incrementarse a medida que se vayan integrando más estudios de especies de plantas de BMM, considerando estacionalidad y estudios moleculares que seguramente revelarán diferentes taxa a los inventarios fúngicos (Wubet, Weiß, Kottke, Teketay, \& Oberwinker, 2006; Kottke et al., 2008; Haug et al., 2010; Lara-Pérez et al., 2014).

A pesar de la baja ocurrencia de HMA en Banderilla (50 esporas como máximo en $10 \mathrm{~g}$ de suelo), se registran niveles de colonización que pueden ser de gran importancia, sobre todo por los bajos niveles de fósforo (P) disponible registrados en este trabajo. Sin embargo, Zongolica presenta una colonización total mayor a pesar que los niveles de $\mathrm{P}$ y nitrógeno $(\mathrm{N})$ fueron considerablemente más altos en comparación con Huatusco y Banderilla. Estos resultados difieren con lo reportado en plantas de interés agrícola donde la colonización micorrízica es mayor cuando el N y P son limitantes (Smith, \& Read, 1997). Se ha sugerido que las plantas que crecen en suelos con baja disponibilidad de N y P tienen que invertir más carbono en la interacción micorrízica para facilitar la translocación de nutrimentos (Smith \& Smith, 2011). Contrariamente, si los niveles de N y P aumentan el grado de colonización se reduce porque los carbohidratos pueden ser asignados 
a otras funciones en la planta (Treseder, 2004). En helechos y licopodios de un ecosistema natural de la isla La Réunion, a través de la presencia-ausencia de colonización, Kessler, Jonas, Strasberg, \& Lehnert (2010) proveen evidencias que indican que la micorriza es una ventaja ecológica especialmente en sustratos deficientes en nutrimentos. En Nueva Zelanda, Cooper (1976) observó que niveles altos de fósforo disponible parece no tener efecto en la colonización de varias familias de helechos incluyendo a helechos arborescentes de la familia Dicksionaceae y Cyatheaceae. Sin embargo, para tener evidencias directas se requiere experimentar con diferentes concentraciones de nutrimentos y medir el grado de colonización micorrízica como lo sugiere Kessler et al. (2010), debido a que solo hay estudios descriptivos y no pueden demostrar directamente el beneficio de la micorriza en helechos y licopodios. Por otra parte, en la inoculación del helecho Rumohra adiantiformis (Dryopteridaceae) con el HMA Glomus intraradices en condiciones controladas en sustrato suplementado con diferentes concentraciones de $\mathrm{N}$ y $\mathrm{P}$, no se afecta la colonización micorrízica (Stamps \& Johnson, 1984).

Dos especies de HMA (Acaulospora scrobiculata, Funneliformis geosporum) estuvieron presentes en los tres sitios con altas abundancias relativas. La presencia de las dos especies de HMA demuestra que tienen amplia distribución y tolerancia a sitios alterados o de alto impacto antrópico como el sitio de Banderilla. Acaulospora scrobiculata tiene amplia distribución en México y está presente en cultivos agrícolas (Aguirre, Carreón, \& Varela 2009; Violi et al., 2008; GuadarramaChávez et al., 2007), vegetación secundaría, selva baja caducifolia (Guadarrama-Chávez, Camargo-Ricalde, Hernández-Cuevas, \& Castillo-Argüero 2007), áreas incendiadas (Violi et al., 2008) y suelos contaminados con petróleo (Franco-Ramírez, Ferrera-Cerrato, VarelaFregoso, Pérez-Moreno, \& Alarcón, 2007). Funneliformis geosporum se encuentra en cultivos agrícolas, ecosistemas naturales en selva baja caducifolia, desiertos y dunas costeras
(Sigüenza, Espejel, \& Allen, 1996; Pezzani, Montana, \& Guevara, 2006; GuadarramaChávez et al., 2007).

Debido a las altas abundancias de esporas de Acaulospora scrobiculata y Funneliformis geosporum pueden ser candidatas para incrementar el inóculo a través cultivos trampa (Brundrett et al., 1996) y probar la eficiencia, solas o en conjunto, en gametofitos y jóvenes esporofitos de $A$. firma.

Aunque no demostramos objetivamente la asociación de las especies de HMA $A$. scrobiculata y $F$. geosporum con A. firma, sus abundancias son considerables en los tres sitios estudiados. Además, su alta tolerancia a sitios alterados puede traer ventajas en su manipulación e inoculación en áreas con manejo, vegetación secundaria o alterada.

A. firma es una de las especies de helecho arborescente que recluta nuevos individuos a la población a través de ramets y de esporas (Riba, 1981; Mehltreter, \& García-Franco, 2008). Estudios de reintroducción de jóvenes esporofitos de $A$. firma (de $5 \mathrm{~cm}$ ) en BMM indican que el borde de bosque es un hábitat apropiado para su establecimiento (Barnabe, Williams-Linera, \& Palacios-Rios, 1999). Eleutério \& PérezSalicrup (2009) registran que individuos jóvenes $<50 \mathrm{~cm}$ de $A$. firma provenientes de hábitats ruderales presenta mayores tasas de supervivencia al ser trasplantado en sombra al $50 \%$ en comparación con sitio expuesto a pleno sol. El alto porcentaje de colonización de arbúsculos de HMA en individuos de $A$. firma indican que es una especie susceptible a la colonización y que podría depender de manera importante de la micorríza. Con la información básica de los requerimientos abióticos de A. firma y el conocimiento de las interacciones ecológicas se contribuirá a mejorar el establecimiento de esta especie. La inoculación con HMA previa a la reintroducción a su hábitat podría conferirle mayor tolerancia a las condiciones adversas, acelerar su crecimiento y reducir la tasa de mortalidad como se ha mostrado en otras especies en estudios anteriores (Gemma, Koske, \& Habte, 2002; Allen, Allen, Egerton-Warburton, 
Corkidi, \& Gomez-Pompa, 2003; Urgiles et al., 2009).

\section{AGRADECIMIENTOS}

El primer autor agradece al Consejo Nacional de Ciencia y Tecnología de México (CONACyT) por la beca otorgada (223899), para realizar estudios de Doctorado en Ciencias en INBIOTECA-UV y a Mauricio D. Valdez Baizabal por el apoyo en campo y laboratorio. A Ricardo Romero, dueño de la reserva Las Cañadas y a Antonio Velázquez y familia por el apoyo logístico en la reserva Amatitla. Se agradece el apoyo financiero de la DGDAIE de la Universidad Veracruzana, gestionado a través de los CAs (UVER-173, UVER- 234 y CA-107).

\section{RESUMEN}

Alsophila firma es un helecho arborescente que crece principalmente en bosque mesófilo de montaña (BMM) y se encuentra en la categoría de riesgo. Los hongos micorrízicos arbusculares (HMA) se han propuesto como una alternativa para programas de recuperación de especies nativas en peligro. Sin embargo, sabemos poco sobre la diversidad de HMA y del estatus micorrízico de las especies vegetales del BMM. En México el BMM presenta diferentes grados de conservación debido a la fragmentación y el cambio de uso de suelo. Se evaluó el grado de colonización, riqueza y abundancia de esporas de los HMA presentes en fragmentos de BMM con diferente historia de manejo: conservado (100 años), en recuperación (17 años) y alterado. Se estudiaron muestras de suelo y raíces tomadas de 5 individuos de $A$. firma por cada sitio, con al menos $100 \mathrm{~m}$ de distancia entre individuos. Se analizaron en total $100 \mathrm{~cm}$ de raíz por cada sitio. Las muestras de raíz presentaron colonización principalmente de HMA y ocasionalmente por hongos septados oscuros (HSO). Se encontraron 19 especies: Gigaspora (7), Acaulospora (4), Glomus (4), Funneliformis (2), Sclerocystis (1) y Scutellospora (1). Las especies dominantes en los tres sitios fueron Funneliformis geosporum y Acaulospora scrobiculata. La mayor diversidad $\left(\mathrm{H}^{\prime}\right)$ y equitatividad $\left(\mathrm{J}^{\prime}\right)(\mathrm{p}<0.05)$ se presentaron en el sitio conservado $\left(H^{\prime}=1.7 ; J^{\prime} 0.66\right)$ con respecto al sitio en recuperación $\left(H^{\prime}=1.5, J^{\prime} 0.61\right)$ y alterado $\left(H^{\prime}=0.74\right.$, $\mathrm{J}=0.41)$. Los niveles de colonización micorrízica total de raíz fueron estadísticamente mayores $(\mathrm{p}<0.05)$ en el sitio conservado. Aunque el sitio alterado presenta una baja riqueza y abundancia de HMA la colonización micorrízica total de raíz no fue estadísticamente diferente $(\mathrm{p}<0.05)$ respecto al sitio en recuperación. El estimador Chao2 indica que las muestras analizadas reflejan la estructura general de la comunidad de HMA con valores superiores al $93 \%$. El presente estudio confirma que $A$. firma es una especie micorrízica con altos niveles de colonización incluso en sitios alterados. Se sugiere que las especies $F$. geosporum y A. scrobiculata por su alta tolerancia a sitios alterados pueden tener potencial para inocular gametofitos y esporofitos de A. firma para incrementar la supervivencia en programas de restauración.

Palabras clave: helecho arborescente, Glomeromycota, número de esporas, índice de diversidad, colonización micorrízica, vegetación alterada, secundaria y conservada.

\section{REFERENCIAS}

Aguirre, P. S., Carreón, A. Y., \& Varela, F. L. (2009). Impacto de la materia orgánica en huertos convencionales y huertos orgánicos de aguacate, sobre la biodiversidad de hongos micorrizógenos arbusculares. Biológicas, 11, 112-121.

Allen, E. B., Allen, M. E., Egerton-Warburton, L., Corkidi, L., \& Gomez-Pompa, A. (2003). Impacts of early- and late-seral mycorrhizae during restoration in seasonal tropical forest, Mexico. Ecological Applications, 13(6), 1701-1717.

Arias, R. M., Heredia-Abarca, G., Sosa, V. L., \& FuentesRamírez, L. E. (2011). Diversity and abundance of arbuscular mycorrhizal fungi spores under different coffee production systems and in a tropical montane cloud forest patch in Veracruz, Mexico. Agroforestry Systems, 85, 179-193.

Bernabe, N., Williams-Linera, G., \& Palacios-Rios, M. (1999). Tree ferns in the interior and at the edge of a Mexican cloud forest remnant: Spore germination and sporophyte survival and establishment. Biotropica, 31(1), 83-88.

Bravo, M. C., \& López, G. A. M. (1999). Inventario de especies vegetales y animales de uso artesanal. Biodiveristas, 22, 9-14.

Brundrett, M. C. (2009). Mycorrhizal associations and other means of nutrition of vascular plants: understanding the global diversity of host plants by resolving conflicting information and developing reliable means of diagnosis. Plant and Soil, 320, 37-77.

Brundrett, M., Bougher, N., Dell, B., Grove, T., \& Malajczuk, N. (1996). Working with mycorrhizas in forestry and agriculture. ACIAR Monograph 32. Canberra, Australia: Australian Centre for International Agricultural Research.

Colwell, R. (2006). EstimateS 8.0b. Statistical estimation of species richness and shared species from simples. Recuperado de http://vivceroy.eeb.uconn.edu. 
Cooper, K. M. (1976). A field survey of mycorrhizas in New Zealand ferns. New Zealand Journal of Botany, 14, 169-181.

Daniels, B. A. \& Skipper, H. D. (1982). Methods for the recovery and quantitative estimation of propagules from soil. In N. C. Shenck (Ed.), Methods and principles of mycorrhizal research (pp. 29-35). St. Paul, Minessotta: American Phytopathological Society.

Dickson, S. (2004). The Arum-Paris continuum of mycorrhizal symbioses. New Phytologist, 163, 187-200.

Eleutério, A. A. \& Pérez-Salicrup, D. (2006). Management of tree ferns (Cyathea spp.) for handicraft production in Cuetzalan, Mexico. Economic Botany, 60(2), 182-186.

Eleutério, A. A. \& Pérez-Salicrup, D. (2009). Transplanting tree ferns to promote their conservation in Mexico. American Fern Journal, 99(4), 279-291.

Franco-Ramírez, A., Ferrera-Cerrato, R., Varela-Fregoso, L., Pérez-Moreno, J., \& Alarcón, A. (2007). Arbuscular mycorrhizal fungi in chronically petroleum contaminated soils in Mexico and the effects of petroleum hydrocarbons on spore germination. Journal of Basic Microbiology, 47, 378-383.

Gemma, J. N., Koske, R. E., \& Flynn, T. (1992). Mycorrhizae in Hawaiian pteridophytes: occurrence and evolutionary significance. American Journal of Botany, 79(8), 843-852.

Gemma, J. N., Koske, R. E., \& Habte, M. (2002). Mycorrhizal dependency of some endemic and endangered Hawaiian plant species. American Fern Journal, 89(2), 337-345.

Gerdemann, J. W. \& Nicolson, T. H. (1963). Spores of mycorrhizal Endogone species extracted from soil by wet sieving and decanting. Transactions of the British Mycological Society, 46, 235-244.

Guadarrama, P., Camargo-Ricalde, S. L., Hernández-Cuevas, L., \& Castillo-Argüero, S. (2007). Los hongos micorrizógenos arbusculares de la región de Nizanda, Oaxaca, México. Boletín de la Sociedad Botánica de México, 81, 131-137.

Hammer, O., D. A. T. Harper \& P. D. Ryan. (2001). PAST (Paleontological Statistics) version. 1.37. Recuperado de http://folk.uio.no/ohammer/past/

Haug, I., Wubet, T., Weiß, M. Aguirre, N., Weber, M. Günter, S., \& Kottke, I. (2010). Species-rich but distinct arbuscular mycorrhizal communities in reforestation plots on degraded pastures and in neighboring pristine tropical mountain rain forest. Tropical Ecology, 51(2), 125-148.

Helgason, T., Daniell, T. J., Husband, R., Fitter, A. H., \& Young, J. P. 1998. Ploughing up the wood-wide web? Nature, 394, 431.
Jumpponen, A. \& Trappe, J. M. (1998). Dark septate endophytes: a review of facultative biotrophic rootcolonizing fungi. New Phytologist, 140, 295-310.

Kaminskyj, S. G. W. (2008). Effective and flexible methods for visualizing and quantifying endorhizal fungi. In Z. A. Siddiqui, M. S. Akhtar, \& K. Futai (Eds.), Mycorrhizae: sustainable agriculture and forestry (pp. 1-18). Dordrecht, The Netherlands: Springer.

Kessler, M., Jonas, R. Strasberg, D., \& Lehnert, M. (2010). Mycorrhizal colonizations of ferns and lycophytes on the island of La Réunion in relation to nutrient availability. Basic and Applied Ecology, 11, 329-336.

Kessler, M., Jonas, R., Cicuzza, D., Kluge, J., Piatek, K., Naks, P., \& Lehnert, M. (2009). A survey of the mycorrhization of Southeast Asian ferns and lycophytes. Plant Biology, 12, 788-793.

Korall, P., Pryer, K. M., Metzgar, J. S., Schneider, H., \& Conant, D. S. (2006). Tree ferns: Monophyletic groups and their relationships as revealed by four protein-coding plastid loci. Molecular Phylogenetics and Evolution, 39, 830-845.

Kottke, I., Beck, A., Oberwinkler, F., Homeier, J., \& Neill, D. (2004). Arbuscular endomycorrhizas are dominant in the organic soil of a neotropical montane cloud forest. Journal of Tropical Ecology, 20,125-129.

Kottke, I., Haug, I., Setaro, S., Suárez, J. P., Weiß, M., Preußing, M., Nebel, M., \& Oberwinkler, F. (2008). Guilds of mycorrhizal fungi and their relation to trees, ericads, orchids and liverworts in a neotropical mountain rain forest. Basic and Applied Ecology, 9, 13-23.

Kubota, M., \& Hyakumachi, M. (2004). Morphology and colonization preference of arbuscular mycorrhizal fungi in Clethra barbinervis, Cucumis sativus, and Lycopersicon esculentum. Mycoscience, 45, 206-213.

Lara-Pérez, L. A. (2007). Efecto de los hongos micorrizógenos arbusculares en la supervivencia de gametofitos micropropagados de Cyathea fulva (Cyatheaceae). (Tesis de Licenciatura). Universidad Veracruzana, Xalapa, Veracruz.

Lara-Pérez, L. A., Noa-Carrazana, J. C., Hernández-González, S., Alarcón-Gutiérrez, E., Sánchez-Velásquez, L. R., Zulueta-Rodríguez, R., Lara-Capistrán, L., \& Andrade-Torres, A. (2014). Diversity and colonization of arbuscular mycorrhizal fungi in the tree fern Alsophila firma in rainy and dry season. Symbiosis, 64(3), 143-150.

Lehnert, M., Kottke, I., Setaro, S., Pazmino, L. F., Suarez, J. P., \& Kessler, M. (2009). Mycorrhizal associations in ferns from Southern Ecuador. American Fern Journal, 99(4), 292-306.

Magurran, A. E. (2004). Measuring biological diversity. Oxford: Blackwell. 
McGonigle, T. P., Miller, M. H., Evans, D. G., Fairchild, G. L., \& Swan, J. A. (1990). A new method which gives an objective measure of colonization of roots of vesicular-arbuscular mycorrhizal fungi. New Phytologist, 115, 495-501

Mehltreter, K. (2010). Interactions of ferns with fungi and animals. In K. Mehltreter, L. R. Walker, \& J. M. Sharpe (Eds.), Fern Ecology (pp. 220-254). UK: Cambridge University Press.

Mehltreter, K. \& García-Franco, J. G. (2008). Leaf phenology and trunk growth of the deciduous tree fern Alsophila firma in a Mexican lower montane forest. American Fern Journal, 98(1), 1-13.

Mehltreter, K., Flores-Palacios, A., \& García-Franco, J. G. (2005). Host preferences of vascular trunk epiphytes in a cloud forest of Veracruz, Mexico. Journal of Tropical Ecology, 21, 651-660.

Mickel, J. T. \& Smith, A. R. (2004). The pteridophytes of Mexico. Memoirs of the New York Botanical Garden, $88,1-1054$

Morton, J. B. (1993). Problems and solutions for integration of glomalean taxonomy, systematic biology, and the study of endomycorrhizal phenomena. Mycorrhi$z a, 2,97-109$.

Moteetee, A., Duckett, J. G., \& Russell, A. J. (1996). Mycorrhizas in the ferns of Lesotho. In J. M. Camus, M. Gibby \& R. J. Johns (Eds.), Pteridology in perspective (pp. 621-631). Kew: Royal Botanic Gardens.

Newsham, K. K., Fitter, A. H., \& Watkinson, A. R. (1995). Arbuscular mycorrhiza protect an annual grass from root pathogenic fungi in the field. Journal of Ecology, 83, 991-1000.

Oehl, F., Sieverding, E., Ineichen, K., Mäder, P., Boller, T., \& Wiemken, A. (2003). Impact of land use intensity on the species diversity of arbuscular mycorrhizal fungi in agroecosystems of Central Europe. Applied and Environmental Microbiology, 69(5), 2816-2824.

Öpik, M., Moora, M., Liira, J., \& Zobel, M. (2006). Composition of root-colonizing arbuscular mycorrhizal fungal communities in different ecosystems around the globe. Journal of Ecology, 94, 778-790.

Öpik, M., Metsis, M., Daniell, T. J., Zobel, M., \& Moora, M. (2009). Large-scale parallel 454 sequencing reveals host ecological group specificity of arbuscular mycorrhizal fungi in a boreonemoral forest. New Phytologist, 184, 424-437.

Ormsby, A., Hodson, E., Li, Y., Basinger, J., \& Kaminskyj, S. (2007). Arbuscular mycorrhizae associated with Asteraceae in the Canadian High Arctic: the value of herbarium archives. Canadian Journal of Botany, $85,599-606$
Pezzani, F., Montana, C., \& Guevara, R. (2006). Associations between arbuscular mycorrhizal fungi and grasses in the successional context of a two-phase mosaic in the Chihuahuan Desert. Mycorrhiza, 16(4), 285-295.

Phillips, J. \& Hayman, D. S. (1970). Improved procedures for clearing roots and staining parasitic and vesiculararbuscular mycorrhizal fungi for assessment of infection. Transactions of the British Mycological Society, $55,158-161$

Riba, R. (1981). Flora de Veracruz; Cyatheaceae. Fasiculo, 17. Xalapa, Veracruz: Instituto Nacional de Investigaciones sobre Recursos Bióticos.

Schenek, N. C. \& Pérez, Y. (1990). Manual for the identification of VA mycorrhizal fungi. Gainesville, Florida, USA: Synergistic Publications.

Schwartz, M. W., Hoeksema, J. D., Gehring, C. A., Johnson, N. C., Klironomos, J. N., Abbott, L. K., \& Pringle, A. (2006). The promise and the potential consequences of the global transport of mycorrhizal fungal inoculum. Ecology Letters, 9, 501-515.

SEMARNAT, (2002). Norma Oficial Mexicana NOM059-ECOL-2001. Diario Oficial de la Federación, México.

Sigüenza, C., Espejel, I., \& Allen, E. B. (1996). Seasonality of mycorrhizae in coastal sand dunes of Baja California. Mycorrhiza, 6, 151-157.

Smith, S. E. \& Read, D. J. (1997). Mycorrhizal symbiosis. San Diego, California: Academic Press.

Smith, S. E. \& Smith, F. A. (2011). Roles of arbuscular mycorrhizas in plant nutrition and growth: new paradigms from cellular to ecosystem scales. Annual Review of Plant Biology, 62, 227-250.

Smith, A. R., Pryer, K. M., Schuettpelz, E., Korall, P., Schneider, H., \& Wolf, P. G. (2006). A classification for extant fern. Taxon, 55(3), 705-731.

Stamps, R. H. \& Johnson, C. R. (1984). Vesicular-arbuscular mycorrhizal inoculation and fertilizer level affect yield, morphology, chlorophyll content, water uptake and vase life of leatherleaf fern frond. Proceedings of the Florida State Horticultural Society, 97, 264-266.

Treseder, M. K. (2004). A meta-analysis of mycorrhizal responses to nitrogen, phosphorus, and atmospheric $\mathrm{CO}_{2}$ in field studies. New Phytologist, 64, 347-355.

Urgiles, N., Lojan, P., Aguirre, N., Blaschke, H., Gunter, S., Stimm, B., \& Kottke, I. (2009). Application of mycorrhizal roots improves growth of tropical tree seedlings in the nursery: a step towards reforestation with native species in the Andes of Ecuador. New Forests, 38, 229-239.

Van der Heijden, M. G. A., Boller, T., Wiekmen, A., \& Sanders, I. R. (1998). Different arbuscular mycorrhizal 
fungi species are potential determinants of plant community structure. Ecology, 76(6), 2082-2091.

Violi, H. A., Barrientos-Priego, A. F., Wright, S. F., Escamilla-Prado, E., Morton, J. B., Menge, J. A., \& Lovatt, C. J. (2008). Disturbance changes arbuscular mycorrhizal fungal phenology and soil glomalin concentrations but not fungal spore composition in montane rain forests in Veracruz and Chiapas, Mexico. Forest Ecology and Management, 254, 276-290.

Vovides, A. P., Luna, V., \& Medina, G. (1997). Relación de algunas plantas y hongos mexicanos raros, amenazados o en peligro de extinción y sugerencias para su conservación. Acta Botánica Mexicana, 39, 1-42.

Wang, B. \& Qiu, Y. L. (2006). Phylogenetic distribution and evolution of mycorrhizas in land plants. Mycorhriza, 16, 299-363.

Williams-Linera, G. (1997). Phenology of deciduous and broadleaved-evergreen tree species in a Mexican tropical lower montane forest. Global Ecology and Biogeography, 6, 115-127.

Wubet, T., Weiß, M., Kottke, I., Teketay, D., \& Oberwinker, F. (2006). Phylogenetic analysis of nuclear small subunit rDNA sequences suggests that the endangered African Pencil Cedar, Juniperus procera, is associated with distinct members of Glomeraceae. Mycological Research, 110, 1059-1069.

Zhao, Z. W. (2000). Study on the relationships between vesicular arbuscular mycorrhiza and the systematic evolution of pteridophyte. Acta Botanica Yunnanica, 22(4), 401-404.

Zubek, S., Piatek, K., Naks, P., Heise, W., Wayda, M., \& Mleczko, P. (2010). Fungal root endophyte colonization of fern and lycophyte species from the Celaque National Park. American Fern Journal, 100(2), 126-136. 
